\title{
Intolerance of Uncertainty and Mindfulness as Determinants of Anxiety and Depression in Female Students
}

Marina Nekić ${ }^{1, *}$ and Severina Mamić ${ }^{2}$

1 Department of Psychology, University of Zadar, Obala kralja Petra Krešimira IV. 2, 23000 Zadar, Croatia

2 Student Counselling Center, University of Zadar, Trg kneza Višeslava 9, 23000 Zadar, Croatia; smamic@unizd.hr

*Correspondence: marina@unizd.hr

\section{Introduction}

Almost $1 / 3$ of college students has symptoms of anxiety and depression (Pirbaglou et al., 2013). Initial results from The WHO World Mental Health Surveys International College Student Project (WHO WMH-ICS) have shown that $35 \%$ of almost 14,000 college students from 19 colleges and 8 countries reports at least one mental disorder

Sociodemographic variables that emerged as being one of the positive correlates of lifetime and 12-month prevalence of mental disorders is female gender (Auerbach et al., 2018).

Academic setting $\rightarrow$ very demanding and challenging for an individual because it emphasizes success and performance (Kurtović, 2013).

Many psychological disorders onset by the age of 24 which overlaps with years spent on college (Kessler, Walters \& Forthofer, 1998).

It is of crucial importance to identify risk factors for the development of psychological disorders among female students. Their identification can lead to development of new techniques and treatments for these disorders.

One factor that emerges as being important for the development of both anxiety and depression is INTOLERANCE OF UNCERTAINTY (IU).

Intolerance of uncertainty is an individual's dispositional incapacity to endure the aversive response triggered by the perceived absence of salient, key, or sufficient information, and sustained by the associated perception of uncertainty.
* transdiagnostic vulnerability factor for the development and maintenance of many psychological disorders (Yook et al., 2010)

$$
\text { WHY? }
$$

IU works as a cognitive bias in terms that the person develops a global negative prediction towards future events (Miranda et al. 2008)

Construct that emerged as one of the protective factors for the development of many psychological disorders $\rightarrow$ MINDFULNESS $\rightarrow$ the state of attention and awareness in the present moment (Brown \& Ryan, 2003).

Shapiro et al. (2006) - intention, attention, and attitude (IAA) are 3 fundamental components of mindfulness which occur simultaneously and lead to shift in perspective they call reperceiving $\rightarrow$ a metamechanism that helps person to disengage from everyday dramas and simply stand back and witness them.

The aim of this study was to determine the contribution of IU and mindfulness in explaining the symptoms of depression and anxiety among female college students.

\section{Materials and Methods}

Participants - 282 female students from different Universities in Croatia of the average age of 21 ( $S D=2,01$; age range: $18-32$ ).

\section{Instruments}

* Intolerance of Uncertainty Scale (NN-11; Mihić et al., 2014)

* Mindful Attention Awareness Scale (MAAS; Brown \& Ryan, 2003)

* Depression, Anxiety and Stress Scale (DASS - 21; Lovibond \& Lovibond, 1995)
Results

\begin{tabular}{|c|c|c|c|c|c|}
\hline Variables & IU & PA & IA & M & A \\
\hline $\begin{array}{c}\text { Intolerance of uncertainty } \\
\text { (IU) }\end{array}$ & & & & & \\
\hline Prospective anxiety (PA) & 0.90 & & & & \\
\hline Inhibitory anxiety (IA) & 0.91 & 0.65 & & & \\
\hline Mindfulness & -0.42 & -0.39 & -0.37 & & \\
\hline Anxiety & 0.43 & 0.41 & 0.38 & -0.43 & \\
\hline Depression & 0.48 & 0.46 & 0.41 & -0.44 & 0.58 \\
\hline
\end{tabular}

IU and mindfulness emerged as reliable predictors of both anxiety and depression among female students. They explained $\mathbf{2 7} \%$ of variance of anxiety, and $31 \%$ of depression.

Mindfulness partially mediated the relation between dimensions of IU and anxiety, and between dimensions of IU and depression.

Individuals' inability to stay calm when faced with uncertainty can predict the onset of anxiety/depression, and this process is partially due to their inability to decenter from negative thoughts and sensations.

It is also possible that mindfulness buffers the influence of Behavioural Inhibition System sensitivity on the development of mental health problems (Hamill et al., 2015).

Result 1 SD below the mean $(\leq 48,86)$ - low on MNDF Result between 48,87 and $69,49-$ moderate levels of MNDF Result 1 SD above the mean $(\geq 69,50)$ - high on MNDF
You want to know how mindful you are? Scan QR code below, fill MAAS and find out your result!
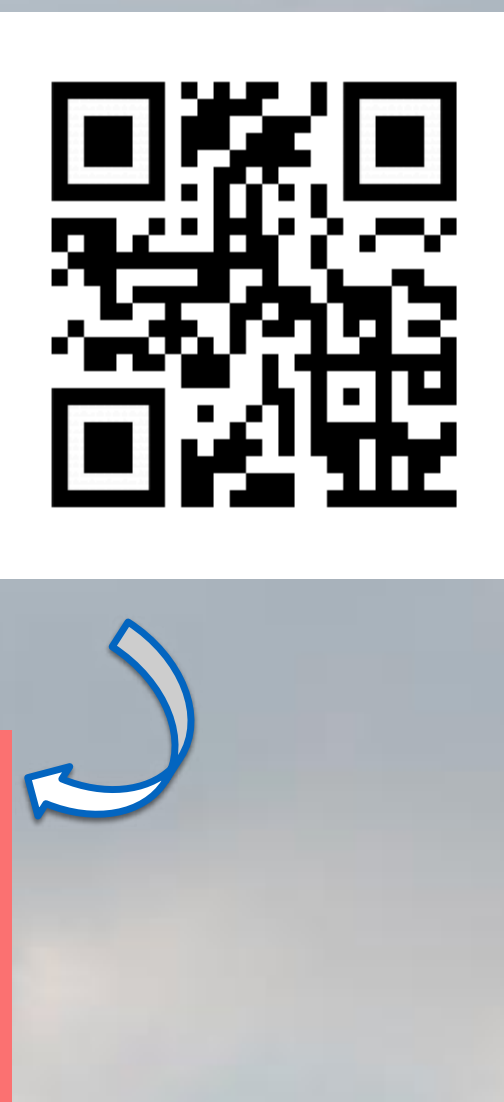